\title{
DESIGN, ADMET AND DOCKING STUDIES ON SOME NOVEL CHALCONE DERIVATIVES AS SOLUBLE EPOXIDE HYDROLASE ENZYME INHIBITORS
}

\author{
KUPPUSAMY ASOKKUMAR ${ }^{a^{*}}$, LOKESWARI TANGELLA PRATHYUSHA ${ }^{a}$, MUTHUSAMY UMAMAHESHWARI ${ }^{a}$, \\ THIRUMALAISAMY SIVASHANMUGAM ${ }^{a}$, VARADHARAJAN SUBHADRADEVI ${ }^{a}$, PULIYATH JAGANNATH ${ }^{a}$, \\ ARUMUGAM MADESWARAN ${ }^{a}$, FRANCIS SALESHEIR ${ }^{b}$
}

\author{
${ }^{a}$ Department of Pharmacology, College of Pharmacy, Sri Ramakrishna Institute of Paramedical Sciences, Coimbatore, India. \\ ${ }^{b}$ Department of Pharmaceutical Chemistry, College of Pharmacy, Sri Ramakrishna Institute of Paramedical Sciences, Coimbatore, India.
} (Received: May 28, 2012 - Accepted: August 10, 2012)

\begin{abstract}
Drug discovery is a lengthy and costly process which aims at bringing in a novel therapeutic molecule for the treatment of various diseases. In the present study, a novel series of eighty chalcone derivatives [(4-substituted)- (4'-substituted)-3' substituted sulphonyl (2E)- 1,3-diphenylprop-2-en-1-one] were designed to inhibit soluble epoxide hydrolase enzyme (sEH). Lipinski's rule of 5 and absorption, distribution, metabolism, elimination and toxicity (ADMET) properties of the compounds were calculated using Molinspiration server and Accord for excel software respectively. All 80 compounds have passed the Lipinski's rule of 5 and only 20 compounds showed considerable ADMET properties. These 20 compounds were subjected to molecular docking studies using AutoDock 4.2 in order to rationalize the possible interactions between test compounds and the active site of human soluble epoxide hydrolase enzyme (1ZD3). Binding energy, intermolecular energy and inhibition constant were the main parameters taken into consideration in this study. The binding energies ranged from -6.07 to -7.89 $\mathrm{kcal} / \mathrm{mol}$, the inhibition constant ranging from $1.64 \mu \mathbf{M}$ to $35.45 \mu \mathrm{M}$ and intermolecular energy ranging between $-9.38 \mathrm{kcal} / \mathrm{mol}$ to $-6.97 \mathrm{kcal} / \mathrm{mol}$. Hence, further pharmacophore optimization and in vivo studies are necessary to develop potent chemical entities that could inhibit the sEH enzyme.
\end{abstract}

Key words: ADMET, docking, human soluble epoxide hydrolase, chalcones, binding energy

\section{INTRODUCTION}

Drug design is an inventive process of finding new medications based on the knowledge of a biological target. Drug discovery and development is an intense, lengthy and an interdisciplinary endeavor. It is a linear, consecutive process that starts with target and lead discovery, followed by lead optimization and pre-clinical in vitro and in vivo studies to determine if such compounds satisfy a number of pre-set criteria for initiating clinical development ${ }^{1-3}$. Present day computational methods deal with all the aspects of drug discovery and have become an essential part of structure-based drug design. They allow developing new drug candidates more rapidly, at a lesser cost. Their key roles in drug discovery are virtual screening and de novo design, in silico ADMET prediction and advanced approaches for defining protein-ligand binding ${ }^{1}$.

Epoxyeicosatrienoic acid (EET) is the epoxide metabolite of arachidonic acid, formed by the action of Cytochrome $\mathrm{P} 450$ epoxygenase in various organs including the liver, kidney and heart ${ }^{4,5}$. They possess many physiologically important roles like fibrinolytic, anti-thrombotic, anti-proliferative, vasodilatory, anti-inflammatory, bronchodilatory and anti-diabetic actions. They also aid in angiogenesis, apoptosis, mitogenesis and secretion of hormones $^{5-7}$. Soluble epoxide hydrolase $(\mathrm{sEH})$ is the primary enzyme involved in the metabolism of chemical mediators ${ }^{6,8,9}$. They hydrolyze EETs to their corresponding dihydroxyeicosatrienoic acid (DHETs). It is suggested that the inhibitors of sEH would effectively increase the in vivo concentration of EETs thereby proving to be useful in the treatment of hypertension, inflammation and other disorders ${ }^{6}$.

Chalcone oxides, trans-3-phenylglycidols, urea and carbamate derivatives were reported to inhibit the enzyme $\mathrm{sEH}^{8,10}$, of which 1,3-disubstituted ureas and carbamates were considered as the most potent and stable inhibitors at nanomolar inhibitory concentrations ${ }^{11-13}$. Therefore in this study, we reported the sEH inhibitory effect of chalcones substituted with the groups like fluoro, chloro, bromo, acetamido and sulphonyl groups at 3' and 4' positions. Special emphases on the ADMET properties of synthesized chalcone derivatives were given to prevent the drug failure at the later phase of drug development.

\section{MATERIALS AND METHODS}

Softwares required

AutoDock 4.2 downloaded from www.scripps.edu, Cygwin, a data storage $\mathrm{C}: \backslash$ program downloaded from www.cygwin.com, Discovery studio viewer 2.5.5 downloaded from www.accelrys.com, MOPAC (Molecular Orbital Package) and Accord for Excel software purchased from Accelrys.inc., ChemDraw 11.0 downloaded from www.acdlabs.com, Molinspiration server and Online Simplified Molecular Input Line Entry System (SMILES) translator were downloaded from http://www.molinspiration.com and cactus.nci.nih.gov/ translate respectively.

Ligand design

Ligands were designed with the basic pharmacophore (NH-CO group) using chalcones as the basic nucleus with different substitutions like fluoro, chloro, nitro, acetamido, methyl, methoxy, hydroxy and sulphonyl substituted secondary amines (morpholine/ N-methyl piperazine/ pyridine/ piperidine) at $4,4^{\prime}, 3^{\prime}$ and $2^{\prime}$ positions. About 80 compounds were designed for $\mathrm{sEH}$ inhibition by incorporating these functional groups. Structure of the ligands was drawn using ChemDraw and SMILES notation was developed for the designed ligands.

Lipinski's Rule of 5

The compounds that were drawn using ChemDraw software were initially screened for Lipinski's rule of 5 using Molinspiration server. The SMILES notation was developed for the structures drawn, which were entered in the tab provided in the online SMILES translator. The option "Calculate of molecular properties and bioactivity prediction" was selected and the properties were calculated $^{14}$.

\section{ADMET properties}

For ADMET properties, initially the structure in ChemDraw were imported into Accord for excel and the options 'ADME functions' followed by the 'ADMET property' were selected and the individual properties were calculated. The ADMET properties so calculated include human intestinal absorption (HIA), hepatotoxicity, CYP2D6 inhibition and blood brain barrier penetration. Chalcone derivatives that have passed the ADMET and Lipinski's rule of 5 were subjected to molecular docking studies using AutoDock 4.2.

Molecular docking

The X-ray crystal structure of sEH complexed with ureido compounds (PDB code 1ZD3) was downloaded from Brookhaeven protein data bank (PDB) and was refined using Accelrys discovery studio visualizer (Figure 1). Using AutoDock Tools, the enzyme, sEH in the .pdb format was converted to .pdbqt format. Then the chalcone derivatives (ligands) which have passed the ADMET tests were optimized for docking studies using "Prepare Ligands" option in the AutoDock 4.2. The grid parameters were set using 'AutoGrid' and the file was saved as .gpf file. By using the Cygwin program the .gpf file was converted into .glg file. The optimized ligand molecules were docked into refined $\mathrm{sEH}$ model using AutoDock 4.2 by setting the algorithm as Lamarckian genetic algorithm and the file was saved as .dpf file which was later converted into .dlg file during the docking process. The dlg file was analyzed using AutoDock Tools and the parameters like binding energy $(\mathrm{Kcal} / \mathrm{mol})$, intermolecular energy $(\mathrm{Kcal} /$ mol) and inhibition constant $(\mu \mathrm{M})$ were considered to select the best docked compounds ${ }^{15,16}$. 




Fig1: Soluble epoxide hydrolase (1ZD3) complexed with a ligand (4-(3-cyclohexylureido)-butyric acid), CU4.

\section{RESULTS AND DISCUSSION}

Lipinski's rule of 5 and ADMET properties

Most drug failures in the drug development process were due to poor pharmacokinetic properties and toxicity. Hence to avoid this, compounds were screened for Lipinski's rule of 5 and ADMET properties. Lipinski's rule of 5 is used to predict the oral bioavailability but not the pharmacological activity. It gives the molecular weight (MW), octanol/water partition coefficient (Log $\mathrm{P}_{\mathrm{o} / \mathrm{w}}$ ), hydrogen bond acceptors (HB- acc), hydrogen bond donors (HB-don) and the number of violations (NOV). Compounds which had a molecular weight of less than 500, hydrogen bond acceptor less than 10, hydrogen bond donor less than 5 , and a $\log \mathrm{P}$ value of less than 5 were considered to be orally bioavailable. According to Lipinski's rule of 5, only two violations are allowed in the molecular docking studies ${ }^{1,2,14}$. Accord for excel was used to calculate ADMET properties like human intestinal absorption (HIA), blood brain barrier penetration (BBB log lev), CYP2D6 inhibition (CYP inh) and hepatotoxicity (Hepatox). Compounds were considered to be hepatotoxic if the value is greater than 0.5 and this was considered as the main parameter to screen the compounds. Compounds with significant ADMET descriptors as well as Lipinski's rule of 5 have been reported in Table 1. Compounds with functional groups like flouro, chloro, nitro and bromo substitutions exhibited hepatotoxicity and these compounds were excluded from our study.

Table 1 Results of ADMET properties and Lipinski's rule of 5 of the designed chalcones.<smiles>[R]c1ccc(C(=O)/C=C/c2ccc([R2])c([R19])c2[R1])cc1</smiles>

BASIC CHALCONE NUCLEUS

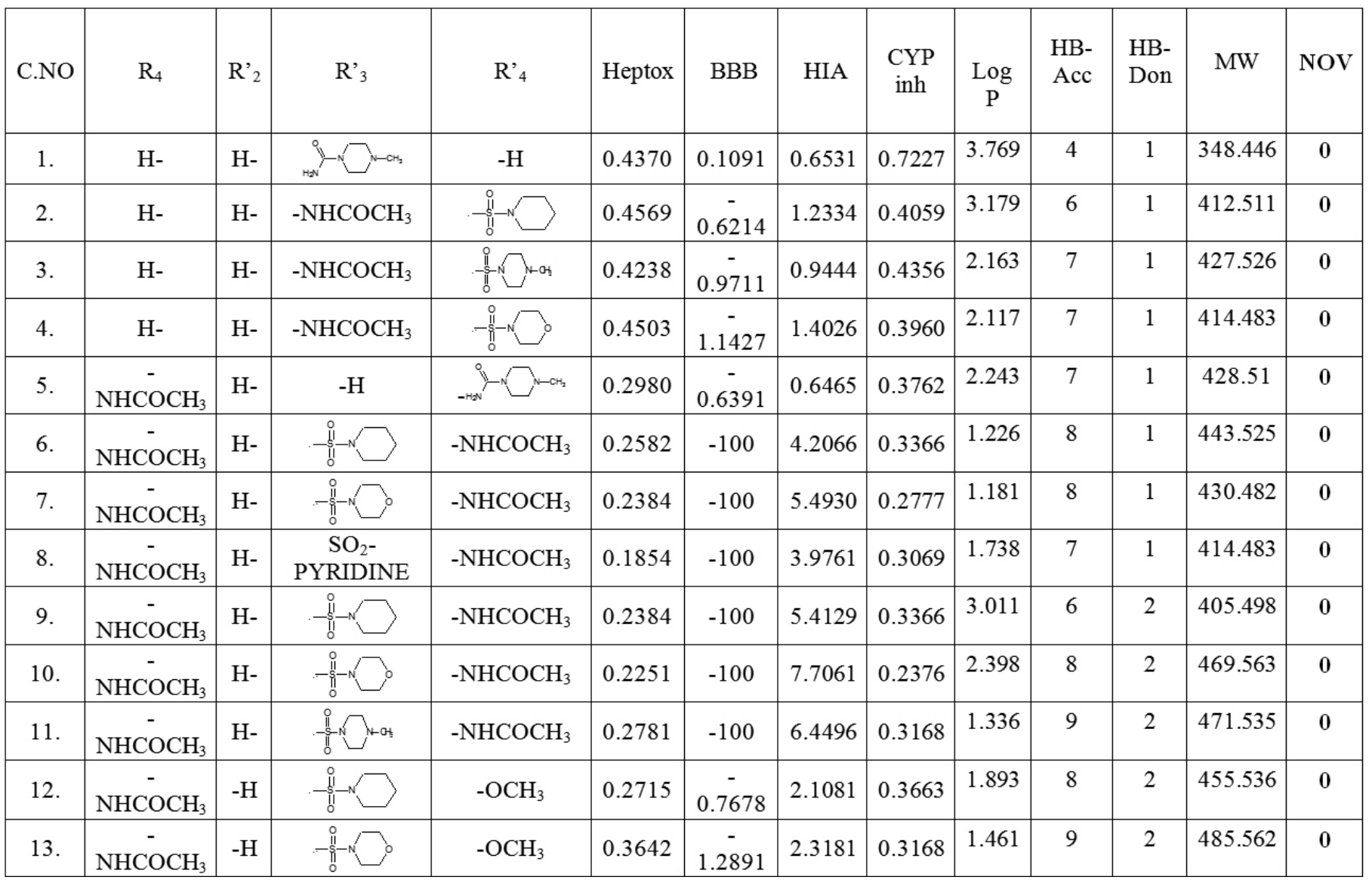




\begin{tabular}{|c|c|c|c|c|c|c|c|c|c|c|c|c|c|}
\hline 14. & $\stackrel{-}{-} \mathrm{NHCOCH}_{3}$ & $-\mathrm{H}$ & & $-\mathrm{OCH}_{3}$ & 0.3311 & $\overline{-}$ & 1.7448 & 0.3366 & 0.399 & 10 & 2 & 487.534 & 0 \\
\hline 15. & $\begin{array}{c}- \\
\mathrm{NHCOCH}_{3}\end{array}$ & $-\mathrm{H}$ & & $-\mathrm{CH}_{3}$ & 0.2847 & $\begin{array}{c}- \\
0.4711\end{array}$ & 1.8946 & 0.2871 & 0.956 & 9 & 2 & 471.535 & 0 \\
\hline 16. & $\begin{array}{c}- \\
\mathrm{NHCOCH}_{3}\end{array}$ & $-\mathrm{H}$ & & $-\mathrm{CH}_{3}$ & 0.3443 & $\begin{array}{c}- \\
0.9924 \\
\end{array}$ & 1.4861 & 0.3168 & 1.163 & 7 & 2 & 456.890 & 0 \\
\hline 17. & $\stackrel{-}{-} \mathrm{NHCOCH}_{3}$ & $-\mathrm{H}$ & & $-\mathrm{CH}_{3}$ & 0.3377 & $\begin{array}{c}- \\
0.8209 \\
\end{array}$ & 1.1155 & 0.3564 & 2.299 & 8 & 1 & 458.536 & 0 \\
\hline 18. & $\begin{array}{c}- \\
\mathrm{NHCOCH}_{3}\end{array}$ & $-\mathrm{H}$ & & $-\mathrm{OH}$ & 0.2847 & -100 & 3.3907 & 0.2277 & 1.237 & 9 & 1 & 460.508 & 0 \\
\hline 19. & $\begin{array}{c}- \\
\mathrm{NHCOCH}_{3}\end{array}$ & $-\mathrm{H}$ & & $-\mathrm{OH}$ & 0.3377 & -100 & 3.8972 & 0.2376 & 1.283 & 9 & 1 & 473.551 & 0 \\
\hline 20. & $\stackrel{-}{\mathrm{NHCOCH}_{3}}$ & $-\mathrm{H}$ & & $-\mathrm{OH}$ & 0.3509 & $\begin{array}{c}- \\
1.3753\end{array}$ & 3.1340 & 0.3663 & 2.023 & 8 & 2 & 444.509 & 0 \\
\hline
\end{tabular}

Molecular Docking

Molecular docking studies help to determine possible interaction of chalcone derivatives with sEH enzyme. The designed compounds were found to have excellent binding affinity to the enzyme. Binding energy of the individual compound was calculated using the following formula,

\section{Binding energy $=\mathbf{A}+\mathbf{B}+\mathbf{C}-\mathbf{D}$}

where, A denotes final intermolecular energy + van der Walls energy $(\mathrm{vdW})+$ hydrogen bonds + desolvation energy + electrostatic energy (kcal/ $\mathrm{mol}), \mathrm{B}$ denotes final total internal energy $(\mathrm{kcal} / \mathrm{mol}), \mathrm{C}$ denotes torsional free energy $(\mathrm{kcal} / \mathrm{mol})$ and $\mathrm{D}$ denotes unbound system's energy $(\mathrm{kcal} / \mathrm{mol})$.

The selected compounds showed binding energy ranging between -6.07 to $-7.89 \mathrm{kcal} / \mathrm{mol}$ which proved that the compounds possess potential $\mathrm{sEH}$ enzyme inhibitory binding sites (Table 2 ).

Table 2. Binding energies $(\mathrm{Kcal} / \mathrm{mol})$ of the chalcone derivatives.

\begin{tabular}{|c|c|c|c|c|c|c|c|c|c|c|}
\hline C.No & $\mathbf{1}^{*}$ & $\mathbf{2 *}$ & $\mathbf{3}$ & $\mathbf{4}$ & $\mathbf{5}$ & $\mathbf{6}$ & $\mathbf{7}$ & $\mathbf{8}$ & $\mathbf{9}^{*}$ & $\mathbf{1 0}^{*}$ \\
\hline 1. & -7.89 & -7.27 & -7.25 & -6.97 & -6.76 & -7.11 & -6.76 & -6.63 & -6.55 & -6.05 \\
\hline 2. & -7.79 & -7.65 & -7.43 & -7.27 & -7.16 & -7.15 & -7.12 & -6.95 & -6.56 & -6.12 \\
\hline 3. & -7.10 & -6.81 & -6.63 & -6.45 & -6.56 & -6.48 & -6.27 & -6.23 & -5.92 & -5.72 \\
\hline 4. & -6.82 & -6.80 & -6.10 & -6.71 & -6.41 & -6.16 & -6.16 & -6.16 & -5.89 & -5.76 \\
\hline 5. & -7.30 & -6.19 & -6.03 & -6.00 & -5.69 & -5.93 & -5.60 & -5.47 & -5.21 & -5.06 \\
\hline 6. & -6.54 & -6.22 & -6.15 & -6.06 & -5.90 & -5.86 & -5.78 & -5.54 & -5.51 & -5.11 \\
\hline 7. & -6.26 & -5.95 & -5.82 & -5.44 & -5.75 & -5.66 & -5.32 & -5.31 & -5.30 & -4.75 \\
\hline 8. & -6.56 & -6.19 & -6.44 & -5.95 & -5.70 & -5.67 & -5.91 & -5.75 & -5.17 & -4.89 \\
\hline 9. & -6.26 & -6.14 & -5.91 & -5.85 & -5.80 & -5.63 & -5.56 & -5.55 & -5.55 & -5.29 \\
\hline 10. & -6.73 & -6.63 & -6.70 & -6.64 & -6.47 & -6.03 & -6.44 & -6.41 & -6.15 & -6.11 \\
\hline 11. & -6.70 & -6.56 & -6.55 & -6.56 & -6.53 & -6.43 & -6.31 & -6.28 & -6.02 & -5.41 \\
\hline 12. & -6.57 & -6.21 & -6.43 & -6.34 & -6.17 & -5.27 & -6.16 & -6.01 & -6.13 & -5.54 \\
\hline 13. & -6.17 & -6.02 & -6.01 & -5.85 & -5.77 & -5.45 & -5.39 & -5.28 & -5.38 & -4.96 \\
\hline 14. & -6.07 & -5.96 & -5.91 & -5.87 & -5.85 & -5.81 & -5.78 & -5.60 & -5.40 & -5.15 \\
\hline 15. & -6.54 & -6.51 & -6.35 & -6.48 & -6.29 & -6.18 & -6.16 & -6.11 & -5.81 & -5.74 \\
\hline 16. & -6.40 & -6.23 & -5.97 & -5.71 & -6.19 & -6.12 & -5.89 & -5.86 & -5.48 & -5.38 \\
\hline 17. & -7.10 & -6.81 & -6.63 & -6.45 & -6.56 & -6.48 & -6.27 & -6.23 & -5.92 & -5.72 \\
\hline 18. & -6.12 & -6.00 & -5.82 & -5.71 & -5.52 & -5.16 & -5.14 & -4.98 & -4.95 & -4.84 \\
\hline 19. & -6.57 & -6.46 & -6.41 & -5.90 & -5.90 & -5.58 & -5.45 & -5.34 & -5.06 & -4.78 \\
\hline 20. & -6.95 & -6.03 & -5.80 & -5.77 & -5.45 & -5.40 & -5.32 & -4.96 & -5.21 & -5.00 \\
\hline
\end{tabular}

$1 *-10 *$ indicate the ten best docked conformations of the compound

In addition, two other parameters like inhibition constant $\left(\mathrm{K}_{\mathrm{i}}\right)$ and intermolecular energy were also determined. Inhibition constant is directly proportional to binding energy. As shown in table 3, the selected compounds showed inhibition constant ranging from $1.64 \boldsymbol{\mu M}$ to $35.45 \boldsymbol{\mu M}$ which implies that they exhibited higher sEH inhibitory activity. 
Table 3. Intermolecular energy (Kcal/mol) of chalcones.

\begin{tabular}{|c|c|c|c|c|c|c|c|c|c|c|}
\hline C.No & $\mathbf{1 *}$ & $\mathbf{2 *}$ & $\mathbf{3}$ & $\mathbf{4}$ & $\mathbf{5}$ & $\mathbf{6}$ & $\mathbf{7}$ & $\mathbf{8}$ & $\mathbf{9}$ & $\mathbf{1 0}$ \\
\hline 1. & -9.38 & 8.76 & -8.75 & -8.47 & -8.25 & -8.60 & -8.25 & -8.12 & -8.04 & -7.54 \\
\hline 2. & -9.58 & -9.44 & -9.22 & -9.06 & -8.95 & -8.94 & -8.91 & -8.74 & -8.35 & -7.91 \\
\hline 3. & -8.89 & -8.60 & -8.42 & -8.24 & -8.34 & -8.27 & -8.06 & -8.02 & -7.71 & -7.51 \\
\hline 4. & -8.61 & -8.59 & -7.89 & -8.50 & -8.20 & -7.95 & -7.95 & -7.95 & -7.68 & -7.55 \\
\hline 5. & -9.09 & -7.98 & -7.82 & -7.79 & -7.48 & -7.72 & -7.39 & -7.26 & -7.00 & -6.85 \\
\hline 6. & -8.33 & -8.01 & -7.94 & -7.85 & -7.69 & -7.65 & -7.57 & -7.33 & -7.30 & -6.90 \\
\hline 7. & -8.35 & -7.98 & -8.23 & -7.74 & -7.49 & -7.46 & -7.70 & -7.54 & -6.96 & -6.68 \\
\hline 8. & -8.35 & -7.98 & -8.23 & -7.74 & -7.49 & -7.46 & -7.70 & -7.54 & -6.96 & -6.68 \\
\hline 9. & -7.45 & -7.33 & -7.10 & -7.04 & -7.00 & -6.82 & -6.75 & -6.75 & -6.74 & -6.49 \\
\hline 10. & -8.23 & -8.12 & -8.19 & -8.13 & -7.96 & -7.52 & -7.93 & -7.91 & -7.64 & -7.63 \\
\hline 11. & -8.49 & -8.35 & -8.34 & -8.35 & -8.32 & -8.22 & -8.10 & -8.07 & -7.81 & -7.20 \\
\hline 12. & -8.65 & -8.30 & -8.52 & -8.42 & -8.25 & -7.36 & -8.25 & -8.10 & -8.22 & -7.62 \\
\hline 13. & -7.06 & -6.93 & -6.90 & -6.74 & -6.66 & -6.34 & -6.29 & -6.17 & -6.28 & -5.86 \\
\hline 14. & -6.97 & -6.85 & -6.81 & -6.77 & -6.75 & -6.70 & -6.67 & -6.49 & -6.30 & -6.04 \\
\hline 15. & -8.04 & -8.01 & -7.84 & -7.97 & -7.78 & -7.67 & -7.65 & -7.60 & -7.31 & -7.23 \\
\hline 16. & -8.49 & -8.32 & -8.06 & -7.79 & -8.28 & -8.20 & -7.98 & -7.95 & -7.57 & -7.47 \\
\hline 17. & -8.89 & -8.60 & -8.42 & -8.24 & -8.34 & -8.27 & -8.06 & -8.02 & -7.71 & -7.51 \\
\hline 18. & -8.20 & -8.09 & -7.91 & -7.80 & -7.60 & -7.25 & -7.23 & -7.07 & -7.04 & -6.93 \\
\hline 19. & -8.66 & -8.54 & -8.50 & -7.99 & -7.99 & -7.67 & -7.54 & -7.42 & -7.15 & -6.87 \\
\hline 20. & -9.04 & -8.12 & -7.89 & -7.85 & -7.54 & -7.49 & -7.41 & -7.05 & -7.03 & -7.09 \\
\hline
\end{tabular}

$1 *-10 *$ indicate the ten best docked conformations of the compound

Intermolecular energy is also directly proportional to binding energy. In our study, there was a decrease in intermolecular energy of all the selected compounds with a simultaneous decrease in the binding energy. The sEH inhibitory activity of the selected compounds was further proved by a decrease in intermolecular energy ranging between $-9.38 \mathrm{kcal} / \mathrm{mol}$ to $-6.97 \mathrm{kcal} / \mathrm{mol}$ (Table 4).

Table 4. Inhibition constant $(\mu \mathrm{M})$ of chalcones.

\begin{tabular}{|c|c|c|c|c|c|c|c|c|c|c|}
\hline C.No & $\mathbf{1}^{*}$ & $\mathbf{2}$ & $\mathbf{3}^{*}$ & $\mathbf{4}^{*}$ & $\mathbf{5}^{*}$ & $\mathbf{6}^{*}$ & $\mathbf{7}^{*}$ & $\mathbf{8}^{*}$ & $\mathbf{9}^{*}$ & $\mathbf{1 0}$ \\
\hline 1. & 1.64 & 4.70 & 4.81 & 7.72 & 11.03 & 6.16 & 11.03 & 13.88 & 15.74 & 36.92 \\
\hline 2. & 1.95 & 2.48 & 3.55 & 4.66 & 5.61 & 5.70 & 6.08 & 8.09 & 15.66 & 32.92 \\
\hline 3. & 6.20 & 10.16 & 13.72 & 18.77 & 15.67 & 17.82 & 25.32 & 26.90 & 45.66 & 63.72 \\
\hline 4. & 10.08 & 10.32 & 33.89 & 12.11 & 20.15 & 30.29 & 30.36 & 30.69 & 47.78 & 60.01 \\
\hline 5. & 4.47 & 29.22 & 37.98 & 39.95 & 67.40 & 44.93 & 79.06 & 97.09 & 152.41 & 194.51 \\
\hline 6. & 16.00 & 27.42 & 31.25 & 36.38 & 47.21 & 50.33 & 58.11 & 87.32 & 91.82 & 178.90 \\
\hline 7. & 25.58 & 43.74 & 54.32 & 102.73 & 61.10 & 71.58 & 126.59 & 128.09 & 130.30 & 332.49 \\
\hline 8. & 15.51 & 29.04 & 18.90 & 43.20 & 66.28 & 69.58 & 46.25 & 60.51 & 161.14 & 259.94 \\
\hline 9. & 25.98 & 31.77 & 46.83 & 51.75 & 55.71 & 75.01 & 84.70 & 84.89 & 85.59 & 131.82 \\
\hline 10. & 11.57 & 13.89 & 12.27 & 13.64 & 18.04 & 38.31 & 19.08 & 19.86 & 30.95 & 33.36 \\
\hline 11. & 12.22 & 15.53 & 15.71 & 15.64 & 16.48 & 19.21 & 23.78 & 24.89 & 38.39 & 108.81 \\
\hline 12. & 15.40 & 27.88 & 19.30 & 22.66 & 30.22 & 137.67 & 30.46 & 39.20 & 32.26 & 87.52 \\
\hline 13. & 30.09 & 38.54 & 39.65 & 51.67 & 59.09 & 101.55 & 11.88 & 135.56 & 113.62 & 230.01 \\
\hline 14. & 35.45 & 42.82 & 46.39 & 49.63 & 51.50 & 55.29 & 58.19 & 79.10 & 109.17 & 168.69 \\
\hline 15. & 15.96 & 16.77 & 22.29 & 17.72 & 24.60 & 29.75 & 30.49 & 33.27 & 54.74 & 62.52 \\
\hline 16. & 20.29 & 26.94 & 41.83 & 65.60 & 28.92 & 32.84 & 48.39 & 50.50 & 95.85 & 112.95 \\
\hline 17. & 6.20 & 10.16 & 13.72 & 18.77 & 15.67 & 17.82 & 25.32 & 26.90 & 45.66 & 63.72 \\
\hline 18. & 32.87 & 39.76 & 53.98 & 65.08 & 90.39 & 163.81 & 169.68 & 224.04 & 235.35 & 283.17 \\
\hline 19. & 15.26 & 18.53 & 19.85 & 47.27 & 47.42 & 81.20 & 101.56 & 122.72 & 196.12 & 312.18 \\
\hline 20. & 8.06 & 37.96 & 56.05 & 59.39 & 101.68 & 110.10 & 125.98 & 231.85 & 152.26 & 214.99 \\
\hline & & & & & & & & & \\
\hline
\end{tabular}

$1 *-10 *$ indicate the ten best docked conformations of the compound 
In figure 2 , the docked pose of chalcone derivatives with $\mathrm{sEH}$ clearly demonstrated the binding positions of the ligand with the enzyme. The main binding force between chalcone derivatives and $\mathrm{SEH}$ is a hydrogen bond between 4'- $\mathrm{NH}$ of acetamido group and the - $\mathrm{COOH}$ of $\mathrm{Asp}^{333}$, which opens the epoxide ring. Other important interactions were found with amino acid residues $\mathrm{Leu}^{513}, \mathrm{Gln}^{335}, \mathrm{Tr}^{334}, \mathrm{Lys}^{514}, \mathrm{Met}^{337}, \mathrm{Gl}^{526}, \mathrm{Val}^{490}$ and $\mathrm{Ala}^{487}$. The compound 1 showed good affinity towards $\mathrm{Asp}^{333}$ binding site which is responsible for better sEH inhibitory activity.

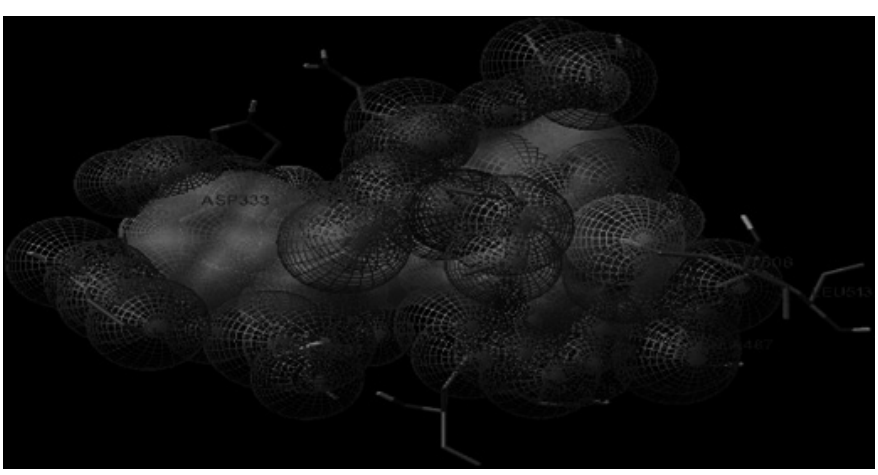

Fig 2: Docked image of the compound $1\left[3^{\prime}\right.$-(N-methyl piperazino) acetamido] 1,3-diphenyl-2- propenone with sEH enzyme.

Gasteiger charges were calculated for each atom of the macromolecule using AutoDock 4.2 instead of Kollman charges which were used in the previous versions of this program. Three-dimensional affinity grids of size 277 $\times 277 \times 277 \AA$ with $0.6 \AA$ spacing was centered on the geometric center of the target protein and were calculated for each of the following atom types: $\mathrm{HD}, \mathrm{C}, \mathrm{A}, \mathrm{N}, \mathrm{OA}$, and SA, representing all possible atom types in a protein. Additionally, an electrostatic map and a desolvation map were also calculated ${ }^{16}$.

On the basis of the above study, it can be proved that chalcone derivatives possess potential sEH inhibitory binding sites which may be attributed due to the differences in the position of the functional groups in the selected compounds.

\section{CONCLUSION}

In the present investigation, different chalcone derivatives were designed, screened for Lipinski's rule of 5 and ADMET properties and the non-toxic compounds were docked to the active site of sEH. The results thus obtained revealed that the substituent with acetamido group on the 4,4' and 3' position of benzene ring of chalcone nucleus may have a considerable impact on the enzyme inhibitory activity of the chalcones. The compounds designed with $-\mathrm{NH}$ and $-\mathrm{CO}$ groups in the basic chalcone nucleus showed more potent inhibitory activity than the previously reported 4-phenyl chalcone oxide. An important factor responsible for the $\mathrm{sEH}$ inhibition is the incorporation of acetamido group which interacts with $\mathrm{Asp}^{333}$ involved in the metabolic pathway of EETs. Hence, further pharmacophore optimization is needed for these analogues to improve their binding affinities and in vivo studies are necessary to develop potent chemical entities that could inhibit the sEH enzyme.

\section{REFERENCES}

1. S. Ekins, Y. Nikolsky, T. Nikolskaya, Trends Pharmacol. Sci. 26, 202 (2005).

2. W.J. Egan, G. Zlokarnik, P.D.J. Grootenhuis, Drug Dis.Today Tech.1, 381 (2004).

3. H. Yu, A. Adedoyin, Drug Disc. Therap. 8, 852 (2003).

4. A.A. Spector, J. Lipid Res. 50, S52 (2009).

5. H. Jiang, A.G. Zhu, M. Mamczur, C. Morisseau, B.D. Hammock, J.R. Falck, J.C. McGiff, J. Pharmacol. Exp. Therap. 326, 330 (2008).

6. U.R. Michealis, I. Fleming, Pharmacol. Theraps.111, 584 (2006)

7. A.A. Spector, X. Fang, G.D. Snyder, N.L. Weintraub, Progress Lip. Res. 43, 55 (2004)

8. K.L. Fife, Y. Liu, K.R. Schmelzer, H.J. Tsai, I.H. Kim, C. Morisseau, B.D. Hammock, D.L. Kroetz, Pharmacol. Exp. Therap. 327, 707 (2008).

9. I.H. Kim, F.R. Heirtzler, C. Morisseau, K. Nishi, H.J. Tsai, B.D. Hammock, J. Med. Chem. 48, 3621 (2005).
10. S. Norwood, J. Liao, B.D. Hammock, G.Y. Yang, Am. J. Transl. Res. 2 , 447 (2010).

11. T. Kasagami, I.H. Kim, H.J. Tsai, K. Nishi, B.D. Hammock, C. Morisseau, Bio. Med. Chem. Let. 19, 1784 (2009).

12. N.R. McElroy, P.C. Jurs, J. Med. Chem. 46, 1066 (2003).

13. Y. Nakagawa, C.E. Wheelock, C. Morisseau, M.H. Goodrow, B.G. Hammock, B.D. Hammock, Bio. Med. Chem. 8, 2663 (2000).

14. C.A. Lipinski, F. Lombardo, B.W. Dominy, P.J. Feeney, Adv. Drug Del. Rev. 46, 3 (2001).

15. G.M. Morris, R. Huey, W. Lindstrom, M.F. Sanner, R.K. Belew, D.S. Goodsell, A.J. Olson, J. Comput. Chem. 19(14), 2785 (2009).

16. A. Madeswaran, M. Umamaheswari, K. Asokkumar, T. Sivashanmugam, V. Subhadradevi, P. Jagannath, In silico docking studies of lipoxygenase inhibitory activity of commercially available flavonoids. Orient Pharm Exp Med 12, 157 (2012). 